\title{
Effect of Suction on Mechanical Behaviour of Unsaturated Soil: A Review
}

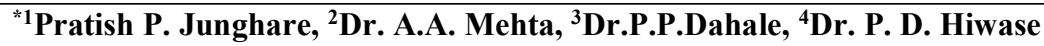 \\ 1,2,3,4 Shri Ramdeobaba College of Engineering and Management, Nagpur \\ Email:1jungharepratish@gmail.com, ${ }^{2}$ mehtaaa@rknec.edu, ${ }^{3}$ dahalepp@rkne.edu, ${ }^{4}$ hiwasep1@rknec.edu
}

Received: 22nd October 2019, Accepted: 20th November 2019, Published: 31st December 2019

\begin{abstract}
This study reviews the mechanical behaviour of unsaturated soil under various Geotechnical problems like bearing capacity failures and the slope stability failures. To obtain a rational solution for any Geotechnical problems, field tests $\&$ numerical modelling were performed. Moreover various empirical equations and new innovating methods used to obtained desire parameters required for unambiguous analytical as well numerical modelling related to unsaturated soil were also discussed. Also, a detailed review on effect of influencing parameters on mechanical behaviour of unsaturated soil is done.
\end{abstract}

\section{Keywords}

Unsaturated Soil, Suction, Factors affecting Shear Strength of Unsaturated Soil.

\section{Introduction}

Now a days significant change in climatic conditions were observed mostly due to Global warming. Many countries experienced either longer rainfall or higher rainfall for shorter time period as rainfall is most frequent triggering factor for slope failure in different parts of world. Moreover the rainwater infiltration and variation in ground water are predominant factor which affected by soil properties. These change in soil pressure responsible for the distinct mechanical behaviour of unsaturated soil under different Geotechnical problems. So the understanding of the mechanical behaviour of unsaturated soil and related parameters which affect the overall behaviour of unsaturated soil is very important. Slope failures in residual soils may occur due to several factors, such as weathering processes, geological activities, human interference, hydrological effect, topographical features, vegetation and climatic conditions. Infiltration of rainwater into soil changes the state of soil from unsaturated to saturated soil. It is well know that negative pore-water pressure which occurs in unsaturated soil increase the shear strength of soil. As soil will gets saturated, Soil suction decreases which finally results in decrease in shear strength. (Fredlund1993)[1]. Fig. 1 represents slope stability failure due to infiltration.

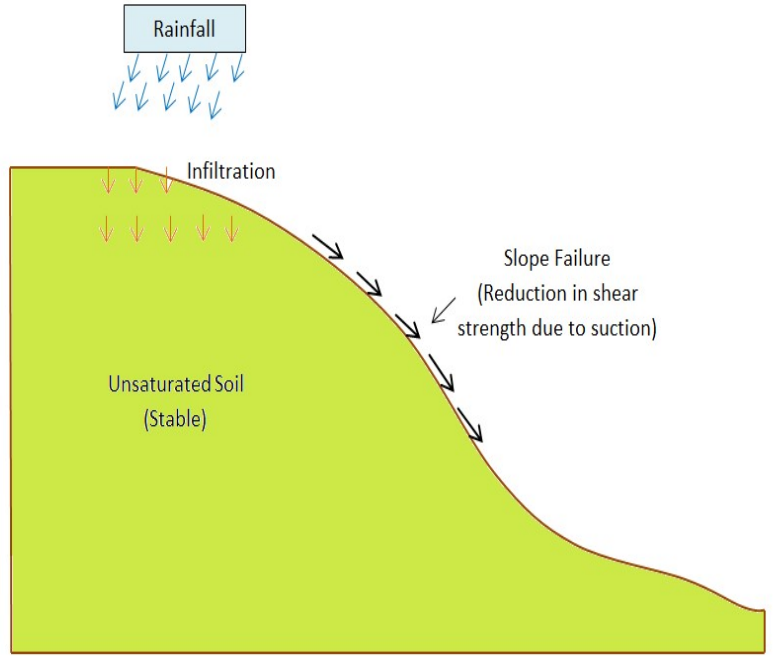

Figure 1: Effect of Rainfall and Suction on Slope Failure

\section{Factors Affecting Mechanical Behaviour of Unsaturated Soil}

It is very useful to understand the unsaturated soil properties, i.e., SWCC required for analysis of seepage or infiltration of water through soil with respect to soil suction. The properties such as shear strength will be required for stability analysis.

\section{Shear Strength}

Unsaturated shear strength of soil is important parameter which affects mechanical behaviour of soil. Higher shear strength contributes to higher FOS against stability of slopes and bearing capacity failures. The shear strength of partially saturated soil is controlled by net normal stress and $\psi$ value [1]. Change in the $\psi$ value due to saturation causes the change in shear strength. A Summary of various empirical equations proposed by previous researchers is shown in table 1. 


\begin{tabular}{ccc}
\hline No. & \multicolumn{1}{c}{ Equation } & Reference \\
\hline 1. & $\tau=\mathrm{c}^{\prime}+\left(\sigma_{\mathrm{n}}-\mathrm{u}_{\mathrm{w}}\right) \tan \varphi^{\prime}$ & Terzaghi[2] \\
\hline 2. & $\tau=\mathrm{c}^{\prime}+\left(\sigma_{\mathrm{n}}-\mathrm{u}_{\mathrm{w}}\right) \tan \varphi^{\prime}+\left(\mathrm{u}_{\mathrm{a}}-\mathrm{u}_{\mathrm{w}}\right) \tan \varphi^{\mathrm{b}}$ & Fredlund et al. [1] \\
\hline 3. & $\begin{array}{c}\left.\tau=\left(\mathrm{c}^{\prime}+\left[\sigma_{\mathrm{n}}-\mathrm{u}_{\mathrm{a}}\right] \tan \varphi^{\prime}\right)+\left[\left(\mathrm{u}_{\mathrm{a}}-\mathrm{u}_{\mathrm{w}}\right)(\Theta \mathrm{k})\left(\tan \varphi^{\prime}\right)\right)\right] \\
\text { OR. }\end{array}$ & Vanapalli et al. [3] \\
\hline 4. & $\tau=\left(\mathrm{c}^{\prime}+\left(\sigma_{\mathrm{n}}-\mathrm{u}_{\mathrm{a}}\right) \tan \varphi^{\prime}\right)+\left(\left(\mathrm{u}_{\mathrm{a}}-\mathrm{u}_{\mathrm{w}}\right)\left(\left(\mathrm{s}_{\mathrm{k}}\right)\left(\tan \varphi^{\prime}\right)\right)\right)$ & Fredlund et al . [1] \\
\hline 5. & $\mathrm{K}=-0.0016 \mathrm{I} 2+0.0975 \mathrm{Ip}+1$ & Vanapalli et al. [3] \\
\hline 6. & $\tan \varphi^{\mathrm{b}}=\left[\mathrm{S}^{\mathrm{k}}\right]\left(\tan \varphi^{\prime}\right)$ & \\
\hline 7. & $\left.\mathrm{C}=\mathrm{c}^{\prime}+\left(\mathrm{u}_{\mathrm{a}}-\mathrm{u}_{\mathrm{w}}\right) \tan \varphi^{\prime}\right)$ & Fredlund et al. [1] \\
\hline 8. & $\mathrm{C}=\mathrm{c}^{\prime}+\mathrm{r}_{\mathrm{c}} \sqrt{\left(\mathrm{s}-\mathrm{S}_{\mathrm{e}}\right) \cdot \mathrm{P}_{\mathrm{a}}}$ & Fredlund et al. [1] \\
\hline 9. & $\mathrm{r}_{\mathrm{c}}=\mathrm{r}_{\mathrm{k}} \frac{\sqrt{3}\left(3-\sin \varphi^{\prime}\right)}{6 \cos \varphi^{\prime}}$ & \\
\hline
\end{tabular}

Table 1: Various Equations Proposed by Previous Researchers for Calculation of Shear Strength of Unsaturated Soil

\section{Bearing Capacity of Unsaturated Soil}

Number of studies shows that the Bearing Capacity in unsaturated soil is affected by soil suction. In many studies the uniform and linear suction distribution is considered for analysing bearing capacity.

\begin{tabular}{|c|c|c|}
\hline Parameter & Linear Suction Distribution & Nonlinear Suction Distribution \\
\hline Cohesion & $\begin{array}{l}\mathrm{C}(\mathrm{z})=\mathrm{C}_{\mathrm{o}}-\lambda \mathrm{z} \\
\mathrm{C}_{\mathrm{o}}=\mathrm{s} \tan \left(\varphi^{\mathrm{b}}\right) \\
\lambda=\frac{\mathrm{s} \tan (\varphi \mathrm{b})}{\mathrm{hw}}\end{array}$ & $\mathrm{c}(\mathrm{z})=\mathrm{s}\left(\frac{\mathrm{hw}-\mathrm{z}}{\mathrm{hw}}\right) \tan \left(\varphi^{\mathrm{b})}\right.$ \\
\hline $\begin{array}{l}\text { Hydrostatic } \\
\text { suction } \\
\text { distribution }\end{array}$ & $\begin{array}{l}\left(\mathrm{q}_{\mathrm{s}}\right)_{\mathrm{hydro}}=\frac{\mathrm{H}}{2 \mathrm{hw}} \mathrm{s} \tan \varphi \mathrm{b}\left(\tan (\alpha)-\mathrm{e}^{\frac{3}{2} \mathrm{xtan} \varphi^{\prime}}+\right. \\
\frac{3}{\cos \alpha} \mathrm{X} \frac{1}{8 \cos ^{2} \varphi^{\prime}-9} \mathrm{X}\left(\mathrm{e}^{\frac{3}{2} \mathrm{xtan} \varphi^{\prime}} \sin \left(\frac{\pi}{4}+\frac{3}{2} \varphi^{\prime}\right)+\right. \\
\cos \left(\frac{\pi}{4}+\frac{3}{2} \varphi^{\prime}\right)+2 \sin \varphi^{\prime}\left(\mathrm{e}^{\frac{3}{2} \mathrm{xtan} \varphi^{\prime}} \cos (\alpha)-\sin (\alpha)\right)\end{array}$ & $\begin{array}{l}\left(\mathrm{q}_{\mathrm{s}}\right)_{\mathrm{hydro}}=\frac{\mathrm{H}}{2 \mathrm{hw}} \operatorname{stan} \varphi \mathrm{b}\left(\tan (\alpha)-\mathrm{e}^{\frac{3}{2} \mathrm{xtan} \varphi^{\prime}}+\right. \\
\frac{3}{\cos \alpha} \mathrm{X} \frac{1}{8 \cos ^{2} \varphi^{\prime}-9} X\left(\mathrm{e}^{\frac{3}{2} \mathrm{xtan} \varphi^{\prime}} \sin \left(\frac{\pi}{4}+\frac{3}{2} \varphi^{\prime}\right)+\right. \\
\cos \left(\frac{\pi}{4}+\frac{3}{2} \varphi^{\prime}\right)+2 \sin \varphi^{\prime}\left(\mathrm{e}^{\frac{3}{2} \mathrm{xtan} \varphi^{\prime}} \cos (\alpha)-\right. \\
\sin (\alpha))\end{array}$ \\
\hline Bearing capacity & $\begin{array}{l}\mathrm{q}_{\mathrm{u}}=\left[\mathrm{c}^{\prime}+\mathrm{c}_{\mathrm{aev}}\right] \mathrm{N}_{\mathrm{c}} \xi_{\mathrm{c}}+\mathrm{qN}_{\mathrm{q}} \xi_{\mathrm{q}}+0.5 \mathrm{~B} \gamma \mathrm{N} \gamma \xi \gamma+\mathrm{sN}_{\mathrm{s}} \tan \varphi^{\mathrm{b}} \xi_{\mathrm{s}} \\
\mathrm{N}_{\mathrm{s}}=\left(\mathrm{N}_{\mathrm{s}}\right)_{1}\end{array}$ & $\begin{array}{l}\mathrm{q}_{\mathrm{u}}=\left[\mathrm{c}^{\prime}+\mathrm{c}_{\mathrm{aev}}\right] \mathrm{N}_{\mathrm{c}} \xi_{\mathrm{c}}+\mathrm{qN}_{\mathrm{q}} \xi_{\mathrm{q}}+0.5 \mathrm{~B} \gamma \mathrm{N} \gamma \xi \gamma^{+} \mathrm{sN}_{\mathrm{s}} \tan \varphi^{\mathrm{b}} \xi_{\mathrm{s}}, \\
\mathrm{N}_{\mathrm{s}}=\left(\mathrm{N}_{\mathrm{s}}\right)_{\mathrm{nl}}\end{array}$ \\
\hline
\end{tabular}

\section{Table 2: Equations for Calculating Bearing Capacity with Linear and Nonlinear Suction Distribution Suction}

A major variation in shear strength value may occur due to suction which finally results in increase in slope stability failures. So, it is important to measures suction variation. Various Direct and Indirect methods of suction measurement is given below: 


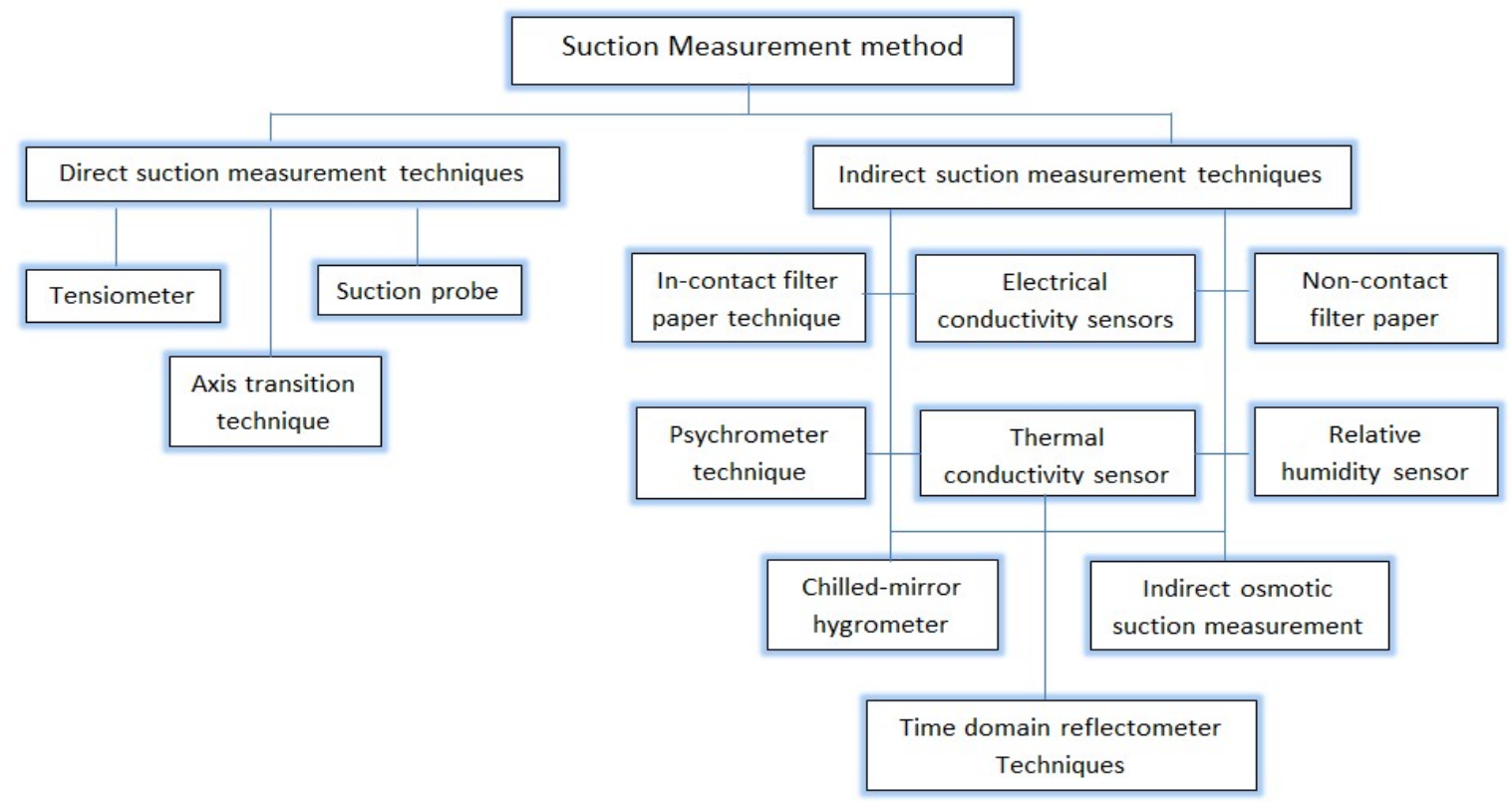

Figure 2: Suction Measurement Techniques.

\begin{tabular}{|c|c|c|c|}
\hline Soil Type & Index properties of soil & Technique used & Reference \\
\hline $\begin{array}{l}\text { Unsaturated } \\
\text { Sand }\end{array}$ & $\begin{array}{l}\text { Particle range: } 0.1 \text { to } 0.5 \mathrm{~mm} \\
\text { Fine Sand }: 7.0 \% \\
\text { Medium Sand }: 93.0 \\
\mathrm{C}_{\mathrm{u}}: 1.20 \\
\mathrm{D}_{10}: 0.21 \mathrm{~mm} \\
\text { Specific gravity }: 2.65\end{array}$ & $\begin{array}{l}\text { Triaxial constant water content test } \\
\text { (CW modified triaxial test) }\end{array}$ & $\begin{array}{l}\text { A. Farouk, L. Lamboj, J. } \\
\text { Kos (2004) [4] }\end{array}$ \\
\hline $\begin{array}{l}\text { Poorly Graded } \\
\text { Sand }\end{array}$ & $\begin{array}{l}\text { Particle Range: } 1 \mathrm{~mm} \text { to } 0.1 \mathrm{~mm} \\
\text { Cu: } 1.5\end{array}$ & $\begin{array}{l}\text { Suction - controlled simple shear } \\
\text { test }\end{array}$ & $\begin{array}{l}\text { Kiyoshi Shimada*; } \\
(1998)[5]\end{array}$ \\
\hline Sandy Clay & $\begin{array}{l}\text { Gs }: 2.62 \\
\mathrm{~W}_{\mathrm{L}}(\%): 39.83 \\
\mathrm{~W}_{\mathrm{P}}(\%): 24.07 \\
\text { Maximum Dry Density, } \\
\text { MDD }(\mathrm{kN} / \mathrm{m} 3) \quad: 15.14 \\
\text { OMC }(\%) ; 23.00 \\
\text { GSA fraction: } \\
\text { \% passing } 75 \text { micron: } 61.39 \\
\% \text { retaining } 75 \text { micron }: 38.61\end{array}$ & $\begin{array}{l}\text { Conventional Triaxial (UU) test. } \\
\text { Soil Suction: Filter paper method. }\end{array}$ & Fredlund et al. [1] \\
\hline Silty Soil & $\begin{array}{l}\text { Fine grained silty soil with zero } \\
\text { cohesion. }\end{array}$ & $\begin{array}{l}\text { Modified conventional triaxial cell. } \\
\text { Soil suction: Pressure plate } \\
\text { apparatus, salt solution desiccators } \\
\text { and relative humidity control } \\
\text { chamber. }\end{array}$ & T. Nishimura, [6] \\
\hline
\end{tabular}




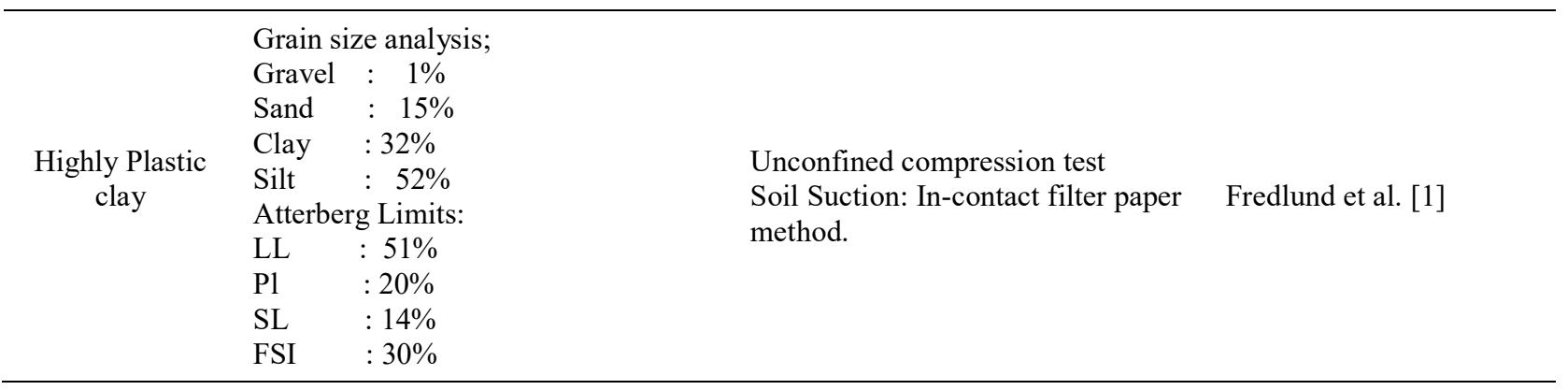

Table 3: Influence of Matric Suction on Shear Strength Behaviour of Various Unsaturated Soil

\section{Soil Water Characteristic Curve (SWCC)}

A Soil water characteristic curves (SWCC) is defined as variation of water content due to matric suction. SWCC is represented graphically as degree of saturation(s), gravimetric moisture content $(\mathrm{w})$, volumetric moisture content $\left(\theta_{\mathrm{w}}\right)$ on ordinate versus matric suction on abscissa in logarithmic scale. The method for estimating SWCC from the AEV which depends on grain size distribution curve which has high Air entry value. Laboratory experiments to obtain SWCC can yield only few data coordinates on varying suction values hence, Fredlund et al. [1] obtained equation using lease-square method as follows;

$$
\theta_{\mathrm{w}}=\mathrm{C}_{(\psi)} \frac{\theta \mathrm{s}}{\left\{l_{n}\left[e+\left(\frac{\psi}{a}\right)^{n}\right]\right\}^{m}} ; \mathrm{C}_{(\psi)}=1-\frac{l_{n}\left(1+\frac{\psi}{\psi \mathrm{r}}\right)}{l_{n}\left(1+\frac{10^{6}}{\psi \mathrm{r}}\right)}
$$

where,

$\theta_{\mathrm{w}}=$ volumetric $\mathrm{w}_{\mathrm{c}}, \theta_{\mathrm{s}}=$ saturated $\mathrm{w}_{\mathrm{c}}, \psi=$ soil suction, $(\mathrm{a}, \mathrm{n} \& \mathrm{~m})=$ fitting correction, $\mathrm{C}_{(\psi)}=$ Correction, and $\psi_{\mathrm{r}}=$ residual water content occurs suction.

\section{Some Sustainable Solution for Slope Stability and Land Slides}

Capillary barrier system consists of layered soil. It is based on well-known principle that the permeability of fine grain soil is lower and coarse grained soil. Figure 3 shows the processing of capillary barrier system in having minimized the infiltration of rainwater into the soil slopes.

While designing the CBS following points will be considered: Rahardjo et al. [7]

i) The passage of water through fine \& coarse grain layers.

ii) Permeability of coarse and fine grained soil.

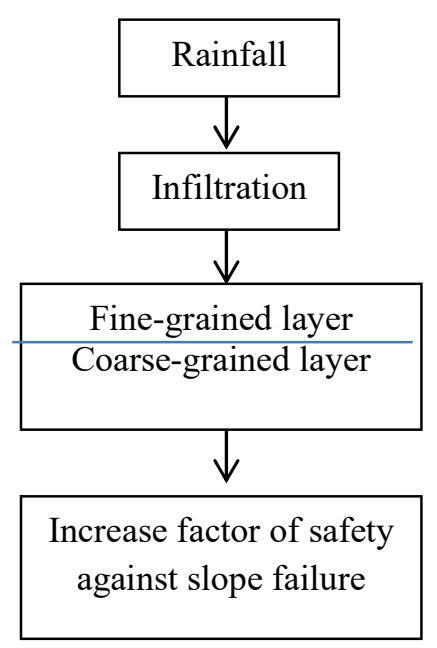

Figure 3: Capillary Barrier System working Mechanism.

Parametric study results showed that vegetation can be used to improve slope stability. The use of type of vegetation depends on various hydrological factors such as soil properties, temperature, evaporation and transpiration rate, rainfall intensity. Generally Vetiver and Orange Jasmine are most commonly used type of vegetation. Vetiver is perianal grass having of fast and deep growing property up to $3.0 \mathrm{~m}$ depth. It has mat-like horizontal spreading system cultivated in tropical area of the world. Orange Jasmine is found in tropical region, can grow up to $7.0 \mathrm{~m}$ tall. It is evergreen plant. However, vegetation contribution is not significant in improving the slope stability as compared to matric suction. 
A. Laboratory Experiment

Proposed by: Technique Used References

Yang et al. (2004) Infiltration test through soil layers were performed using onedimensional (1-D) soil column.

Yang, H., Rahardjo, H., Wibawa, B. and Leong. E.C. (2004) [8]

$\begin{array}{ll}\text { Tami et al. (2004a) } \quad \text { Residue water Infiltration } & \text { Fredlund et al. [1] }\end{array}$

Indrawan(2006) Use of various soil mixtures in fine-layers of CBS

Krisdani et al. (2006) Use of geosynthetic material with proper gradation

B. Field Experiments $\begin{array}{ll}\text { Rahardjo (2010) } & \begin{array}{l}\text { Measured pore water pressure using tensiometers and } \\ \text { piezometers }\end{array}\end{array}$
Yang, H., Rahardjo, H., Wibawa, B. and Leong. E.C. (2004) [8]

Yang, H., Rahardjo, H., Wibawa, B. and Leong. E.C. (2004) [8]

Yang, H., Rahardjo, H., Wibawa, B. and Leong. E.C. (2004) [8]

Table 4: Effects of Capillary Barrier System on Various Properties of Soil

\section{Conclusion}

As above stated parameters adversely affects the slope failures and bearing capacity failures of unsaturated soil, an engineer should have a keen knowledge about all these parameters. To fulfil these requirements new innovative methods studied by previous researchers were reviewed to obtained sustainable solution of related geotechnical problems.

\section{References}

1. D.G. Fredlund, \&Rahardjo, (1993) "Unsaturated Soil Mechanics". New York.

2. Terzaghi K, The shear strength of saturated soils, Proceedings of the First International Conference on Soil Mechanics and Foundation Engineering, Cambridge, MA, 1936, pp.54-56.

3. Vanapalli SK, Fredlund DG, Pufahl DE, and Clifton AW, Model for the prediction of shear strength with respect to soil suction, Can. Geotech. J., 1996, pp. 379-392.

4. Farouk, L. Lamboj, J. Ko, Influence of Matric Suction on the Shear Strength Behaviour of Unsaturated San, Czech Technical University Publishing House, ActaPolytechnica Vol. 44 No. 4/2004.

5. Kiyoshi Shimada*, (Received October 31, 1997, Effect of Matric Suction on Shear Characteristics of Unsaturated Fraser River Sand, Journal of the Faculty of Environmental Science and Technology, Okayama University Vol.3, No.1, pp.127-134, January 1998.

6. T. Nishimura (Ashikaga Institute of Technology, Japan), Relationship between shear strength and matric suction in an unsaturated silty soil, Conference on Unsaturated soils, UNSAT ASIA 2000 Singapore, May 18-19, 2000

7. Rahardjo, H., Hua, C.J., Leong, E.C. and Santoso, V.A. (2010) "Performance of an Instrumented Slope under a Capillary Barrier System”. Proc. 5th International Conference on Unsaturated Soils, Barcelona, Spain, 6-8 September, 2, pp1279-1284.

8. Yang, H., Rahardjo, H., Wibawa, B. and Leong. E.C. (2004) “A Soil Column Apparatus for Laboratory Infiltration Study”. Geotechnical Testing Journal, ASTM International, July, 27, Issue 4, pp347 - 355. 